\title{
EDGE VERSUS CONTRAST ESTIMATION OF MORPHOLOGICAL FILTERS
}

\author{
P. Salembier(1), J. Serra ${ }^{(2)}$ and J.A. Bangham ${ }^{(3)}$ \\ (1): Dep. of Signal Theory and Com., Universitat Politècnica de Catalunya, Barcelona, Spain. \\ (2): Centre de Morphologie Mathématique, Ecole des Mines de Paris, Fontainebleau, France. \\ (3): School Information Systems, University of East Anglia, Norwich, UK.
}

\begin{abstract}
This paper deals with the estimation properties of morphological filters in terms of edge localization and grey level contrast preservation in two dimensional spaces. It is shown that, at least in practice, a compromise between these two characteristics has to be done. In a first step, morphological filters are compared with linear and median filters. It is concluded that, an efficient edge localization can be achieved with morphological filters with reconstruction and not with median filters or with morphological openings or closings with structuring elements. In the case of strong noise, the good edge preservation provided by the reconstruction filters is obtained at the expense of the contrast estimation. In order to be able to tune this tradeoff, a new type of morphological filters, called filters with partial reconstruction, are proposed and studied.
\end{abstract}

\section{INTRODUCTION}

The goal of this paper is to investigate the performance of morphological filters in terms of edge and contrast estimation. This study comes from the classical statement following which nonlinear filters are very good at localizing edges. In the case of one dimensional signal, the study reported in [1] has investigated the edge and amplitude estimation properties of linear and median filters. This study concludes that a tradeoff exists between those two parameters and that the median filter is actually very good at localizing edges if it is used within a multiresolution structure. The multiresolution approach means that, instead of filtering the signal directly with a filter of window size $n$, one should use compositions of filters with increasing window size from one to $n$.

The present study concentrates on the case of morphological and median filters for two dimensional signals. Note that the results for median filters in 2D cannot be directly extrapolated from those of $1 D$ at least for the following reason: in 1D, median filters preserve edges because their root (invariant) signals are pulses. In $2 \mathrm{D}$, this is not true and for example a square is not a root signal of a median filter with a squared window. Therefore, its edges are not preserved by the filtering. This problem was quoted in [2] where edge preservation of median filters as well as morphological filters are studied. This study concludes that median or morphological filters such as opening, closing or open_close filters are not very good for $2 \mathrm{D}$ edge preservation even if they are used within a multiresolution structure. However a "reconstruction" process which dramatically improves the edge preservation properties was used. Finally, it was mentioned that the grey level contrast of the image may not be preserved by the reconstruction process. This remark leads to the tradeoff discussed in [1].

By contrast with [2], which has a qualitative approach, this paper precisely quantifies the edge and grey level contrast estimation characteristics of morphological and median filters for various noise probability density functions (Laplacian, Gaussian and uniform). Special attention is paid to the problem of multiresolution structures to see when it is useful. Finally, the results motivate the definition of a new class of morphological filters based on partial reconstruction.

The organization of this paper is as follows: the next section presents the basic notions involved in morphological signal processing and filtering. Then, section 3 is devoted to the quantitative assessment of the filters performances.

\section{MORPHOLOGICAL FILTERS}

This section briefly describes the morphological notions of interest for this study. More details can be found in [3]. The first part of the presentation is devoted to basic notions and structures. Then, the second part deals with morphological filters which will be studied in section 3 .

\subsection{Basic notions}

By contrast with linear signal processing which is based on the mathematical structure of vectorial space, mathematical morphology relies on a structure called complete lattice [5] Basically, a complete lattice is a set of elements on which an ordering relation ( $\leq$ ) has been defined. Moreover, each family of elements $\left\{X_{i}\right\}$ possesses a supremum (maximum lower bound) and an infimum (minimum upper bound). This study is concerned with input signals which are discrete grey level functions. The corresponding lattice relies on an order which is simply defined at each spatial position by the natural ordering between grey level values: two functions $f, g$ are such that $f \leq g$ if for each spatial position $x, f(x) \leq g(x)$. Moreover, in the case of discrete signal, the two laws, infimum and supremum, are respectively minimum and maximum.

On this lattice, the first transformations to be defined are those preserving the structure, that is the ordering relation $\leq$, and commuting with one of the two laws. Note that this procedure is similar to that used in linear signal processing. In this last case, the basic structure is a vectorial space where the fundamental laws are the addition between two vectors and the scalar product. The first transformation to be defined is a transformation which preserves the space, that is which transforms a vector into a vector, and which commutes with the fundamental laws, that is:

$$
\psi(\alpha f+\beta g)=\alpha \psi(f)+\beta \psi(g)
$$

It turns out that the only transformation fulfilling eq.(1) is the convolution. In mathematical morphology, the same approach leads to increasing transforms, erosions and dilations:

Preserve of the structure: $\quad f \leq g=>\psi(f) \leq \psi(g)$

These transformations are called increasing 
Commute with Max: $\quad \psi(\operatorname{Max}\{f, g\})=\operatorname{Max}\{\psi(f), \psi(g)\}$ (3)

These transformations are called dilations

Commute with Min: $\quad \psi(\operatorname{Min}\{f, g\})=\operatorname{Min}\{\psi(f), \psi(g)\} \quad(4)$

These transformations are called erosions

These equations lead to an infinity of erosions and dilations. For the sake of simplicity, only two examples of dilation (and erosion) are presented in the sequel:

\section{Dilation and erosion with flat structuring element:}

If $f\left(x_{i}\right)$ denote a signal and $M_{n}$ a window or flat structuring element of size $n$, the erosion and dilation are given by:

Erosion: $\quad \varepsilon_{n}(f)\left(x_{i}\right)=\operatorname{Min}\left\{f\left(x_{i}+k\right), k \in M_{n}\right\}$

Dilation: $\quad \delta_{n}(f)\left(x_{i}\right)=\operatorname{Max}\left\{f\left(x_{i-k}\right), k \in M_{n}\right\}$

More general definitions of erosions and dilations with non flat structuring element can be found in the literature [3]. Here, flat structuring elements are preferred because they

- preserve edges: this will be discussed in section 3 .

- allow fast implementation: with flat structuring element, it is possible to define algorithms for the erosion and the dilation leading to a computation time which is independent of the size of the structuring element.

- are robust with respect to grey level changes: in particular, they commute with modifications of the grey level scale with functions such as $\log$ or square root.

These two transformations are dual in the sense that:

$$
\delta_{\mathrm{n}}(\mathrm{f})=-\varepsilon_{\mathrm{n}}(-\mathrm{f})
$$

\section{Geodesic grey level dilation and erosion:}

The geodesic transforms [4] of a given function are always defined with respect to a reference function (section 2.2 will discuss the choice of this reference). The geodesic dilation of size one (that is the smallest size on the discrete space) is defined as the minimum between the dilation of size one of the original function (f) and the reference function ( $r$ ). The geodesic erosion is defined by duality:

Geodesic dilation and erosion of size one:

$\delta(1)(f, r)=\operatorname{Min}\left(\delta_{1}(f), r\right\}$

$$
\varepsilon(1)(f, r)=-\delta(1)(-f,-r)
$$

The justification of this terminology involving the notion of geodesy goes beyond the scope of this description and the reader is referred to [4] for more details. Geodesic dilations and erosions of large size are defined by iterations. For example, the geodesic dilation (resp. erosion) of infinite size, also called reconstruction by dilation (resp. erosion) is given by:

Reconstruction by dilation and erosion:

$$
\gamma^{(\mathrm{rec})}(\mathrm{f}, \mathrm{r})=\delta^{(\infty)}(\mathrm{f}, \mathrm{r})=\ldots \delta^{(1)}\left(\ldots \delta^{(1)}(\mathrm{f}, \mathrm{r}) \ldots, \mathrm{r}\right)
$$$$
\varphi^{(\mathrm{rec})}(\mathrm{f}, \mathrm{r})=\varepsilon^{(\infty)}(\mathrm{f}, \mathrm{r})=\ldots \varepsilon^{(1)}\left(\ldots \varepsilon^{(1)}(\mathrm{f}, \mathrm{r}) \ldots, \mathrm{r}\right)
$$

Note that the reconstruction can be implemented very efficiently, in particular, avoiding any iterating process [5].

\subsection{Morphological filters}

A morphological filter is any increasing and idempoten transformation. In addition, if the transformation is antiextensive (resp. extensive), it is called an algebraic opening (resp. algebraic closing). Let us recall that: $\psi$ is idempotent if for all $\mathrm{f}, \psi(\psi(\mathrm{f}))=\psi(\mathrm{f})$

(9)

$\psi$ is extensive if for all $\mathrm{f}, \mathrm{f} \leq \psi(\mathrm{f})$

$\psi$ is antiextensive if for all $\mathrm{f}, \psi(\mathrm{f}) \leq \mathrm{f}$

\section{Morphological opening and closing:}

The most classical cases of opening and closing are those based on the composition of erosion and dilation with structuring element. They are called morphological:

$$
\begin{array}{ll}
\text { Morphological opening: } & \gamma_{n}=\delta_{n} \varepsilon_{n} \\
\text { Morphological closing: } & \varphi_{n}=\varepsilon_{n} \delta_{n}
\end{array}
$$

The opening (resp. closing) simplifies by removing the bright (resp. dark) components which do not fit within the structuring element. If the simplification has to deal with both bright and dark elements an open_close $\left(\gamma_{n} \varphi_{D}\right)$ or a close_open $\left(\varphi_{n} \gamma_{n}\right)$ has to be used. None of these filters are self-dual (dual of itself), but in practice they approximately remove the same kind of information. Fig. 1 presents examples of filtering results with open_close filter together with linear and median filters. It clearly shows that none of these filters perfectly preserve contour information as discussed in [2]. To improve the contour preservation, reconstruction processes can be used.

\section{Opening and closing by reconstruction.}

The opening (resp. closing) by reconstruction of erosion (dilation) is defined as:

Opening by rec. of erosion: $\gamma^{(\mathrm{rec})}\left(\varepsilon_{\mathrm{n}}(\mathrm{f}), \mathrm{f}\right)$

Closing by rec. of dilation: $\varphi^{(\mathrm{rec})}\left(\delta_{\mathrm{n}}(\mathrm{f}), \mathrm{f}\right)$

Let us take the first filter as an example: $\gamma^{(\mathrm{rec})}\left(\varepsilon_{\mathrm{n}}(\mathrm{f}), \mathrm{f}\right)$. The simplification is performed by the erosion which eliminates all signal components that are smaller than the structuring element. Then, the reconstruction process restores the contour of the components which have not been totally removed by the erosion. Note that the reference function ( $r$ ) mentioned in 2.1 is $\mathrm{f}$ itself. The effect of an opening by reconstruction followed by a closing by reconstruction can be seen in Fig. 2.a where the very good contour preservation property is clearly visible. The resulting image is much simpler than the original image, but the objects that are present are precisely defined.

In fact, these filters can be generalized as follows: from any algebraic opening $(\gamma)$, an opening by reconstruction can be defined by thresholding: let $X_{t}(f)$ denote the binary signal resulting from the thresholding of $f$ at a grey level value of $t$. Consider now, for each $t$, the connected components $Y_{t}(f)$ of $\mathrm{X}_{\mathrm{t}}(\mathrm{f})$ containing at least one point of $\mathrm{X}_{\mathrm{t}}(\gamma(\mathrm{f}))$. Then, the opening by reconstruction is defined as the grey level function obtained by stacking the $Y_{t}(f)$ for all $t$. It can be demonstrated that this new transform is an algebraic opening. In practice, the reconstruction process is implemented by geodesic dilation of infinite size of $\gamma(f)$ with $f$ as reference (eq. (8)):

Opening by reconstruction: $\gamma^{(\mathrm{rec})}(\gamma(\mathrm{f}), \mathrm{f})$

Closing by reconstruction: $\varphi^{(\mathrm{rec})}(\varphi(\mathrm{f}), \mathrm{f})$

In the case of strong noise, it may not be appropriate to reconstruct totally the contour of the objects since they are highly corrupted. In this case, the reference signal for the reconstruction process, that is f, may be smoothed by a small 
opening $\gamma_{k}(f)$. This approach leads to a new class of morphological filters called filters with partial reconstruction.

$$
\begin{aligned}
& \text { Opening by partial rec.: } \gamma^{(\mathrm{rec})}\left(\varepsilon_{\mathrm{n}}(\mathrm{f}), \gamma_{\mathrm{k}}(\mathrm{f})\right) \\
& \text { Closing by partial rec.: } \varphi^{(\mathrm{rec})}\left(\delta_{\mathrm{n}}(\mathrm{f}), \varphi_{\mathrm{k}}(\mathrm{f})\right)
\end{aligned}
$$

Note that if $k=n$, these filters correspond respectively to the morphological opening and closing, whereas if $k=0$, they become the opening and closing by reconstruction. Fig. 2 presents the results obtained with an opening followed by a closing with partial reconstruction. The parameter $\mathbf{k}$ allows a smooth tuning of the reconstruction process from no reconstruction $(k=n)$ to "full" reconstruction $(k=0)$.

\subsection{Multiresolution filtering}

These filters can be used within a multiresolution structure which states that instead of filtering with a filter of size n, one should use composition of filters of increasing size from one to $n$. In the case of open_close, this composition is known as alternating sequential filters. Let $M_{n}$ denote a filter involving a window of size $\mathbf{n}$ (for example a square window encompassing $\mathrm{n}^{*} \mathrm{n}$ pixels), the multiresolution filter is given by:

Multiresolution filter: $M_{n}\left(M_{n-1} \ldots M_{k}\left(\ldots M_{1}().\right)\right)$

\section{RESULTS}

\subsection{Criterion}

To assess the relative quality of each filter, a synthetic image can be used. It is composed of several objects on a background and it has been corrupted by noise. The advantage of using a synthetic image is that the optimal edge localization and grey level value estimation results are a priori known. The image is first filtered and then segmented (several segmentation algorithms have been used. They give similar results). Once the image has been segmented, two parameters are measured:

- The normalized number of pixels differing between the actual and the optimal segmentation. This parameter is referred to as Edge Estimation. It essentially measures the property of contour preservation of the filter.

- The mean variance of the grey level differences between the inside of each segmented region and the background. This parameter, called Contrast Estimation, assesses the filter efficiency to preserve grey level differences.

The contrast criterion is used because most of the filters of interest are not self-dual and give a biased estimation of the absolute grey level value. However, in very few applications the absolute grey level value is of interest (it requires an extreme and often unrealistic control of the processing chain from the illumination and acquisition to the final display). What is more generally required is that the difference between two grey level values is preserved or can be precisely estimated. This feature is the contrast criterion used here.

Each measure is plotted on a bidimensional plane (Edge / Contrast). In fact, for each filter, a set of measures are obtained by modifying the window (or structuring element) size. This creates a curve in the Edge / contrast plane. Finally, several tests have been performed with various noise probability density functions such as Laplacian, Gaussian and uniform. As far as the criteria described above are concerned, the Gaussian case leads to intermediate results between the Laplacian and uniform cases. Therefore, only the results obtained with Laplacian and uniform noise are presented here.

\subsection{Experimental results}

The experimental results are reported on Fig. 3 to 4 . They respectively represent the contrast versus the edge estimation in the case of Laplacian and uniform noise. Each curve represents the performances of a given filter when its window size changes. The best filters are characterized by a curve which is close to the origin of the plane.

In the case of Laplacian noise, the best filter in terms of contrast estimation is the median filter. The morphological open_close filter does not provide better results. As expected, the linear filter does not give a good estimation of the edge location and its contrast estimation performances are close to that of the median filter. Both, median and morphological open close filters achieve similar performances (better than the linear filter) in terms of edge localization. However, the open_close by reconstruction improves dramatically the edge preservation properties. Multiresolution filtering improves the performances mainly in terms of robustness with respect to the window size. There is no major change in the absolute performances. This observation contrasts with the results reported in [1] where multiresolution filtering proved to be much better than direct filtering. The results reported here are typical of multidimensional signals and can be explained by the different root properties observed for 1 and 2D nonlinear filters (see introduction). For example, the corners of a triangle are not preserved by a median or a morphological filter with a squared window.

In the case of uniform noise, the results are quite similar except that the open_close is better than the median filter for contrast estimation (in the case of Gaussian noise, both filters give equivalent results). As can be seen, the open_close filter has good contrast estimation properties whereas the open_close with reconstruction is better for edge estimation. This observation leads to the use of morphological filters with partial reconstruction to achieve a compromise between edge and contrast preservation. Fig. 4 presents an example of such a filter where the partial reconstruction was obtained by setting $k=n-1$ in eq.(16). This filter has very attractive characteristics for applications where both good edge and contrast preservation is required.

\section{REFERENCES}

[1] J.A. Bangham, T.G. Campbell, M. Gabbouj, The quality of edge preservation by non-linear filters, In Proc. of the IEEE Workshop of Visual Signal Processing and Communications, Rayleigh, pp. 36-39, 1992.

[2] P. Salembier and M. Kunt, Size-sensitive multiresolution decomposition of images with rank order based filters, in Signal Processing, Vol.27, No2, pp.205-241, 1992.

[3] J. Serra, Image Analysis and Mathematical Morphology, Academic Press, Vol. I-1982, Vol. II-1988.

[4] C. Lantuéjoul and F. Maisonneuve, Geodesic methods in image analysis, in Pattern Recognition, Vol. 17, 1984.

[5] L. Vincent, Algorithmes morphologiques a base de files d'attente et de lacets, Ext. aux graphes, PhD Thesis, 1990. 


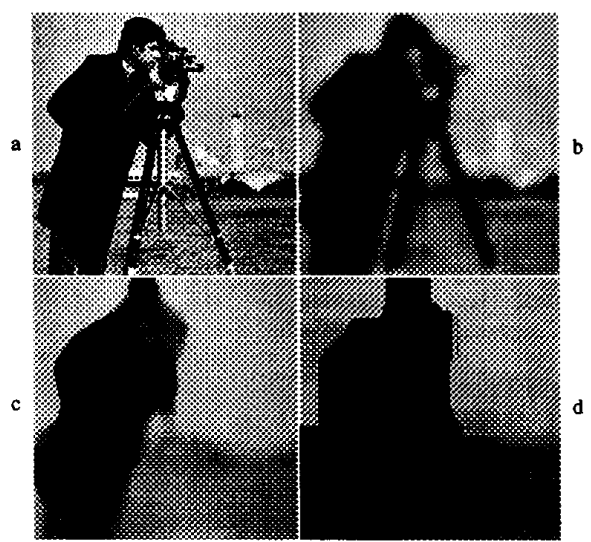

Figure 1: Example of filtering a: Original, b: Linear filter c: Median filter, d: Open_close

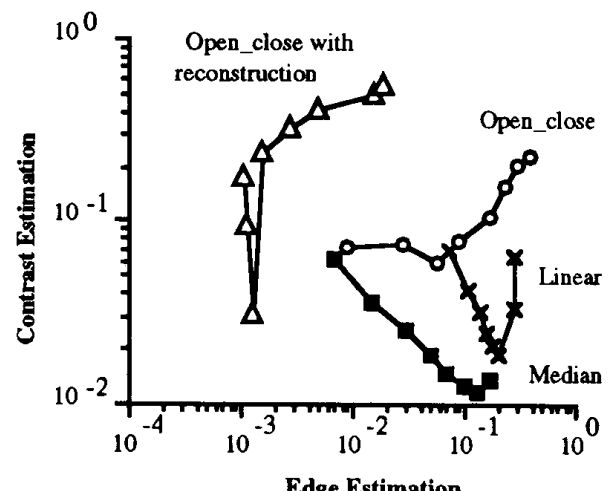

Figure 3.a: Edge versus contrast estimation of filter (Laplacian noise, $S N R=25 \mathrm{~dB}$ ), direct filtering

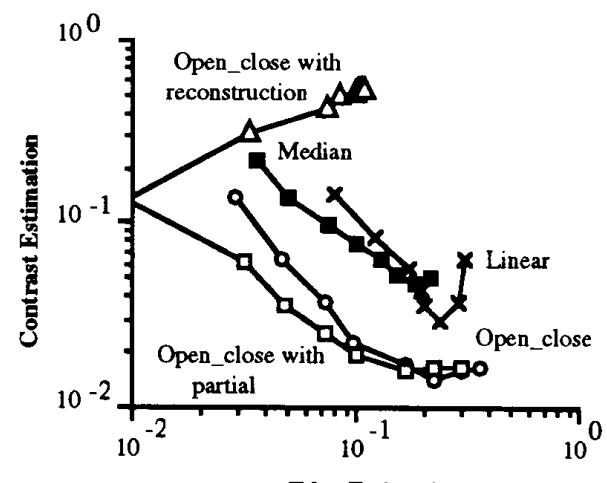

Edge Estimation

Figure 4.a: Edge versus contrast estimation of filter (IID noise, $S N R=18 \mathrm{~dB}$ ), direct filtering

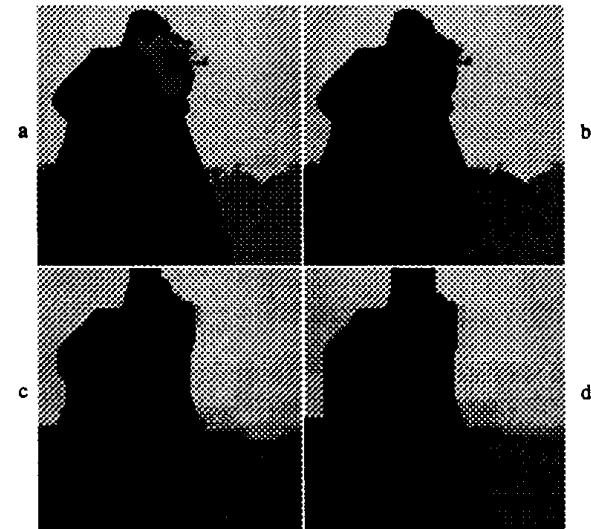

Figure 2: Filtering with reconstruction a: Full recons., b: Partial rec. $k=1$

c: Partial recons. $k=n / 2$, d: No recons. $k=n$

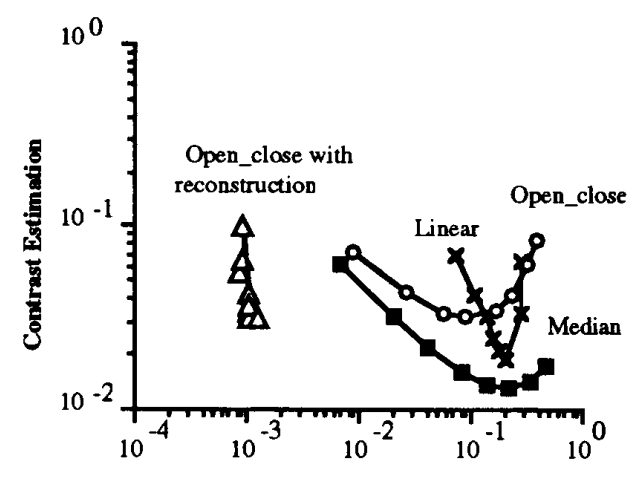

Figure 3.b: Edge versus contrast estimation of filter (Laplacian noise, $S N R=25 \mathrm{~dB}$ ), multires.filtering

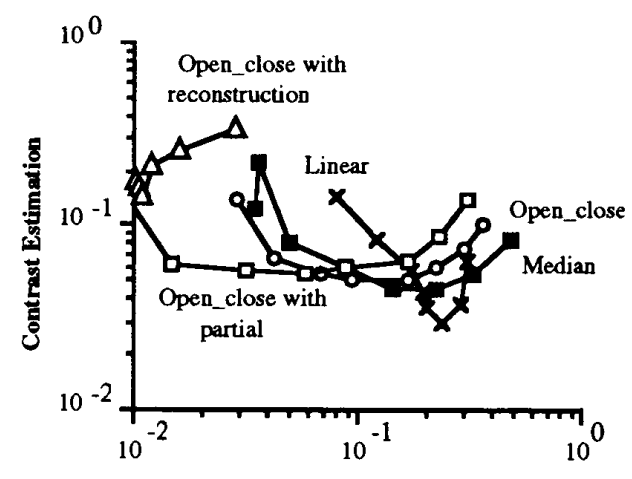

Edge Estimation

Figure 4.b: Edge versus contrast estimation of filter (IID noise, $S N R=18 \mathrm{~dB}$ ), multiresolution filtering 